\title{
Interdisciplinary Treatment for a Compensated Class II Partially Edentulous Malocclusion: Orthodontic Creation of an Implant Site
}

\author{
Grace Chiu, Chris Chang, W. Eugene Roberts
}

\begin{abstract}
Diagnosis: A 36 year-old female presented with good periodontal health, and a compensated Class II partially-edentulous malocclusion, associated with a steep mandibular plane (SN-MP $45^{\circ}$ ), nine missing teeth, a $3 \mathrm{~mm}$ midline discrepancy, and compromised posterior occlusal function. There were multiple carious lesions, a failing fixed prostheses in the lower right (LR) quadrant replacing tooth \#30, and a severely atrophic edentulous ridge in the areas of teeth \#18-19 in the lower left (LL) quadrant.
\end{abstract}

Treatment: After restoration of the caries, the LL third molar (\#17) served as anchorage to correct lower arch crowding. The second premolar $(\# 20)$ was retracted with a light force of $20 z(\sim 28.3 \mathrm{cN})$ on the buccal and

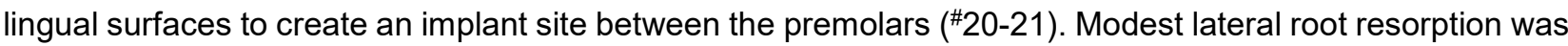
noted on the distal surface of $\# 20$ following $~ 7 \mathrm{~mm}$ of distal translation in $7 \mathrm{mo}$. Six months later, LR and LL implants were placed and the spaces were retained with the fixed appliance for $5 \mathrm{mo}$ and a removable retainer for $1 \mathrm{mo}$. Poor cooperation resulted in the relapse of \#20 back into the implant site, and it was necessary to reopen the space. When the LL fixture was uncovered, there was about a $3 \mathrm{~mm}$ deep osseous defect on the distobuccal surface, which was an area of relatively immature bundle bone, because the distal aspect of the space was reopened after the relapse. Subsequent bone grafting resulted in good osseous support of the implant-supported prosthesis, the relative thin band of attached gingiva on implant $\# 30$ healed with a recessed contour, that was susceptible to food impaction. A free gingival graft restored soft tissue form and function.

Outcomes: This severe malocclusion with a Discrepancy Index (DI) of 28 was treated to an excellent outcome with 38 months of interdisciplinary treatment, as evidenced by a Cast-Radiograph Evaluation (CRE) score of 13. However, the treatment was complicated by routine relapse and implant support problems. Retreatment of space opening, plus two additional surgeries were required to correct an osseous defect and an inadequate soft tissue contour.

Conclusion: Orthodontics is a viable option for creating implant sites, but fixed retention is required until the prosthesis is delivered. Bone augmentation is indicated at the time of implant placement to offset expected bone loss. Complex restorative treatment may result in routine complications that are effectively managed with interdisciplinary care.

\section{Introduction}

Permanent teeth may be lost to trauma, caries, or periodontal disease. There is a marked reduction in width and height of the alveolar ridge following tooth extraction,,$^{1,2}$ that often compromises prosthetic restoration. ${ }^{3}$ Preprosthetic ridge augmentation with on-lay bone grafting is often required prior to or in conjunction with implant placement. ${ }^{4}$ If the periodontium is healthy, orthodontic site development is a viable alternative for producing new bone and attached gingiva. ${ }^{5-9}$ Movement of a healthy tooth through an edentulous area to increase the ridge dimensions to receive an implant is a promising method, but there is a risk of lateral root resorption. ${ }^{7}$ Orthodontic tooth movement is capable of expanding the alveolar process, ${ }^{3,7,8}$ and placing

This is the author's manuscript of the article published in final edited form as:

Chiu, G., Chang, C., \& Roberts, W. E. (2018). Interdisciplinary treatment for a compensated Class II partially edentulous malocclusion: Orthodontic creation of a posterior implant site. American Journal of Orthodontics and Dentofacial Orthopedics, 153(3), 422-435. https://doi.org/10.1016/j.ajodo.2016.11.029 
implants in these sites has been suggested, ${ }^{5-9}$ but to the authors' knowledge there are no documented reports for implant placement in orthodontically generated sites in posterior segments. The current case report describes routine complications associated with orthodontically generated implant sites, and how these minor complications are solved in an efficient manner. An excellent functional and esthetic result was achieved, as documented by a Cast-Radiograph Evaluation (CRE) score of 13.

\section{Diagnosis and Etiology}

A 36 year-old female sought consultation for space management in the lower left posterior area (Figs. 1-3), and was treated to an excellent dentofacial result (Figs. 4-6). She was diagnosed with a skeletal Class I and a compensated dental Class II with a missing left maxillary canine (Fig.1-3). The etiology of the complex malocclusion was unrestorable caries and the extraction of the lower left first and second molars ( $* 18$ and 19), about 7 years previously (Fig. 7). The adjacent third molar (*17) drifted mesially and tipped into the space, resulting in an atrophic edentulous space that was about $14 \mathrm{~mm}$ in length (Figs. 3 and 7). Clinical and radiographic evaluations revealed multiple carious lesions and a failing LR fixed prosthesis replacing tooth *30 (Figs. 2 and 7). In addition, the lower left central incisor was missing, resulting in a lower midline deviation of $\sim 3 \mathrm{~mm}$ to the left (Figs. 2 and 3). The patient reported that the right first premolar ("5) and the left canine (*11) were extracted by her family dentist when she was 13 years of age because they were blocked-out to the buccal (Fig. 2). The upper right second molar ("2) was missing (unknown etiology) and the adjacent third molar had drifted into the second molar space. The American Board of Orthodontics (ABO) Discrepancy Index (DI) ${ }^{10}$ was 28 points as shown in the subsequent Worksheet 1. The implant sites ( $\# 19$ and 30 ) scored an additional 4 points for complexity (see supplement to Worksheet 1). Overall, this mutilated malocclusion was a severe problem requiring a carefully sequenced, interdisciplinary approach. The post-treatment radiographs (Fig. 8) and superimposed cephalometric tracings (Fig. 9) documented the interdisciplinary treatment.

\section{Treatment Objectives}

1. Restore all carious lesions

2. Maintain the skeletal dimensions of the maxilla and mandible for all three planes.

3. Preprosthetic tooth movement:

- Protract and upright $\# 17$ to reduce the edentulous space (Figs. 10 and 11)

- Retract \#20 to the position of \#19 and place an implant in the space opened between \#20 and 21 (Figs. 12-15)

4. Prosthetics:

- Remove the lower right fixed prosthesis and restore teeth \#29 and 31 with crowns

- Place a dental implant-supported prosthesis in the edentulous ridge of area \#30

5. Soft Tissue Management:

- When uncovered, evaluate each implant for an apically repositioned flap or free gingival graft to increase the buccal keratinized tissue and soft tissue volume

6. Orthodontics Finishing: Optimize dentofacial esthetics with orthodontic detailing

\section{Treatment Alternatives}


The first alternative for restoring the LL edentulous area was to augment the ridge and place two implants. However, that approach required an additional implant, and was less predictable because of the complexity of the surgery (surgeries) to augment the severe bone atrophy of the implant site. In addition, the mesial inclination of \# 17 (Fig. 7) risked long term periodontal problems, periodontitis and/or peri-implantitis, due to food impaction and plaque accumulation between \#17 and the distal implant. The second option was a removable partial denture to replace the LL missing molars, but the mesial inclination of \# 17 was expected to encroach on the path of denture insertion. That problem would require the extraction of \#17, or restorative procedures to prepare it for a surveyed crown. The patient rejected both alternative treatment approaches because of additional surgery, potential for the longterm compromise of the virgin tooth \#17, and increased risk of periodontal problems.

\section{Treatment Progress}

A full fixed Damon $\mathrm{Q}^{\circledR}$ appliance (Ormco, Glendora, CA) was used with the archwires and accessories specified by the manufacturer. Mechanics began in the lower arch with an .014" NiTi archwire, and five months later an .014x.025" NiTi archwire was inserted. Lingual buttons and power chains were placed on teeth \#22 and 20 to control rotation. In the $7^{\text {th }}$ month of treatment, the lower archwire was switched to $.017 x .025$ " TMA. Another lingual button and power chain were placed on $\# 17$ to correct mesial rotation and provide a mesial protraction force (Fig. 10). By the end of the $16^{\text {th }}$ month of treatment, all rotations were corrected and the edentulous space was reduced to $8 \mathrm{~mm}$ (Fig. 11), but the width of the edentulous ridge was still narrow $(\sim 3 \mathrm{~mm})$ due to severe resorption of the buccal plate of bone (Fig. 12). Tooth \#20 was retracted orthodontically to generate an implant site between the lower left premolars ( $\# 20$ and 21). Buccal and lingual force was applied as: 1 . an open coil spring placed on the archwire between teeth $\# 20$ and 21 , and 2 . lingual buttons with a power chain between teeth \#20 and 17 (Fig. 12). The force was applied very gently, $\sim 2 \mathrm{oz}(28.3 \mathrm{cN})$ on each surface to control lateral root resorption. After 7 months of retracting $\# 20$ at a rate of $1 \mathrm{~mm} / \mathrm{mo}$, the orthodontically generated implant site was around $7 \mathrm{~mm}$ in length (Fig. 14). The space was maintained for 6 months with the fixed appliances. A periapical radiograph revealed only minor lateral root resorption on the distal side of the root of \#20 (Fig. 15).

Extraction of blocked-out permanent teeth ( $\# 2,5$ and 11) during adolescence corrected crowding in the upper arch, so only minor orthodontic treatment was needed to correct the mesial-in rotation of both lateral incisors (Fig. 16). After bonding with the Damon $Q^{\circledR}$ appliance, interproximal reduction was performed on teeth \#7-10 to create space. The archwire sequence was .016" NiTi, .016x.022" NiTi, and .016x.022" SS to align the dentition (Fig. 16). After 32 months of active treatment, both arches were well aligned and the bilateral edentulous spaces in the lower first molar areas were prepared for implants (Figs. 17a and b).

Implant placement

A horizontal incision was made with a No. 12 blade from the lingual line angle of tooth ${ }^{\#} 21$ to the mesial line angle of ${ }^{\# 20}$ (moved into the ${ }^{\# 19}$ position). Two vertical releasing incisions were made with a No. 15 blade and joined with the horizontal incisions. The incisions were $1.5 \mathrm{~mm}$ away from the adjacent teeth for maintenance of the periodontal attachment to control gingival recession post-operatively (Fig. 17c). A full thickness flap was reflected, and a surgical gauge measured the $6 \mathrm{~mm}$ wide ridge (Figs. $17 \mathrm{~d}$ and $\mathrm{e}$ ). A surgical stent was used to check the projected soft tissue height (Fig. 17f), and the crestal bone was re-contoured with a round bur (Fig. $17 \mathrm{~g}$ ) to create $3 \mathrm{~mm}$ clearance to the bone crest, as required for the biologic width of the attachment (Fig. 17h). ${ }^{11}$ A guide-pin (Fig. 17i) was used to check the insertion path and orientation of the osteotomy (Fig. 17j). The osteotomy site was prepared according to the protocol of the manufacturer and a $3.5 \times 10 \mathrm{~mm}$ implant was installed (Prima, Keystone Dental Inc., Burlington, MA). As shown in Figures 17j and k, the implant was placed in the center of the ridge and was completely embedded (submerged) in bone. Furthermore, about a $1.5 \mathrm{~mm}$ 
thickness of bone remained on the buccal surface after implant placement (Fig. 17j). There was primary closure of the wound with interrupted 5-0 Nylon sutures (Fig. 17-I).

A similar procedure was performed on the lower right edentulous ridge in the area of \#30 (Fig. 18a-h). However the ridge was narrower in the crestal area and after implant placement, there was $1 \mathrm{~mm}$ bony dehiscence noted at buccal crest of the implant (Fig. 18-f). The healing abutment was installed, and a composite particulate bone graft was placed (Fig. 18-g), that was composed of FDBA (freeze-dried bone allograft) produced by Maxxeus Dental, Kettering $\mathrm{OH}$, and Bio-Oss $®$ which is marketed by Geistlich Pharma North America, Princeton NJ. The flap was then primarily closed with 5-0 Nylon. FDBA is composed of cortico-cancellous human bone granules that serve as a scaffold for osteoconduction, but the graft material is eventually remodeled to living bone. BioOss $®$ is a preparation of sterile, dense bovine cancellous granules that also serves as a scaffold for bone deposition, but the graft material is turned over to bone very slowly.

Both wounds healed uneventfully, and the arch spaces were maintained with a plastic sheath on the right side (Fig. 19a) and an open-coil spring on the left (Fig. 19b). After 6 months of stabilization with no activation, all fixed appliances were removed and clear overlay retainers were fabricated for both arches. Cooperation with the lower overlay retainer was inadequate and in one month tooth ${ }^{*} 20$ relapsed to the mesial (Fig. 20). Fixed appliances were re-applied to the lower arch with power chains and open coil springs to correct the relapse (Fig. 21). After five months of re-treatment, tooth ${ }^{\#} 20$ was repositioned in a well aligned arch.

The total healing time for the LL implant was about 12 months, including the post-operative relapse of the space opening, along with the follow-up orthodontics to reopen the space. Implant uncovering was accomplished with horizontal and vertical incisions made with No. 12 and 15 blades, respectively. An apically positioned flap was planned to move the keratinized tissue on the crest to the buccal aspect of the implant, but when the full-thickness flap was reflected, an osseous defect was revealed on the distobuccal surface of the fixture, that exposed $>2 \mathrm{~mm}$ of rough implant surface (Fig. 22b). The stability of the implant was carefully evaluated, and there was no mobility. A healing abutment was placed and a particulate bone graft of FDBA +Bio-Oss $₫$ as previously described was applied on the buccal surface to cover the exposed portion of the implant. The wound was closed with 5-0 Nylon. Two months after the second surgery, all fixed appliances were removed and clear overlays were again provided to retain both arches. It was emphasized to wear the clear overlays for 24 hours a day for the first three months and nights only thereafter. In addition, a fixation wire was bonded between teeth \#20 and 18 to prevent reopening of the space. A screw-retained, final implantsupported prosthesis was fabricated and installed on the implant (Fig. 22f).

\section{Free gingival graft}

The lower right implant-supported prosthesis was completed according to the standard protocol, and functioned normally, but the patient complained that food was often impacted on the buccal surface. Clinical examination revealed a $0.5 \mathrm{~mm}$ width of keratinized gingiva that was associated with a deep concave depression in the distal cervical area adjacent to the buccal vestibule (Fig. 23). A free gingival graft as illustrated in Figure 24 was required in the right posterior segment to increase the soft tissue thickness, contour and width of the keratinized gingiva. A horizontal incision was made apical to the attached gingiva (24-a) from teeth \#29-31, and a partial thickness flap was reflected (Fig. 24-b). The periosteum was left intact as the recipient bed for a free gingival graft (Fig. 24-c). Prior to the grafting procedure, the donor site was covered with platelet rich fibrin (PRF), ${ }^{12}$ which was prepared by drawing venous blood from the patient. The blood was centrifuged and decanted under sterile conditions, leaving the fibrin matrix with platelets, cytokines, and growth factors (Fig 24-d). A 5x15mm free gingival graft was harvested from the right side of the palate (Fig. 24-C). All adipose tissue was removed and the graft was sutured over the recipient site with 5-0 nylon. A Coe-Pak ${ }^{\circledR}$ (GC America, Alsip, IL) dressing was applied to protect the graft and to prevent the buccal flap from reattaching to 
the recipient bed. Six months later, the free gingival graft was well integrated and the objectives were achieved: increased soft tissue thickness, improved contour and a wider band of keratinized gingiva (Fig. 25). The concave gingival area was corrected with non-mobile keratinized tissue, that deflected the flow of masticated food into the vestibule, thereby reducing buccal impaction.

\section{Treatment Results}

Overall, the dental and facial esthetics as well as masticatory function were well managed (Figs. 4-6). The ABO Cast-Radiograph Evaluation (CRE) $)^{13}$ score was 13 points, as shown in the subsequent Worksheet 2. The only significant detraction from the excellent CRE score was buccal tipping and insufficient occlusal contacts on the upper left quadrant (Figs. 5 and 6). That problem was associated with the missing left maxillary canine. Achievement of ideal interdigitation and optimal posterior occlusal contacts is a common problem when a compensated occlusion lacks a maxillary canine.

The severe atrophic ridge (area ${ }^{\# 19)}$ was successfully developed from 3 to $7 \mathrm{~mm}$ with orthodontic space opening. With light forces, ${ }^{\#} 20$ was translated distally at a relatively rapid rate of $1 \mathrm{~mm} / \mathrm{mo}$, and the only adverse effect was slight lateral root resorption (Fig. 15) on the distal root where it engaged the most dense aspect of the knife edge ridge (Figs. 11 and 12). Occlusal function was considerably improved by restoring both lower first molars with implant-supported prostheses (Figs. 5, 6 and 8). The design of an apically positioned flap and free gingival graft on the lower right buccal surface (Fig. 25) successfully increased the keratinized gingiva and improved the long term stability of the soft tissue supporting the implant-supported prosthesis. The increased tissue volume and improved gingival curvature improved the flow of food during mastication, which reduced the prevalence of buccal food impaction.

\section{Discussion}

Orthodontic tooth movement into edentulous areas is a viable option for providing a prosthesis abutment or enhancing the ridge dimensions to serve as an implant site..$^{3,7} \mathrm{~A}$ longitudinal study with an average follow-up of 9.6 years revealed the orthodontically moved teeth were well maintained with excellent gingival conditions but there was a slight marginal bone loss. ${ }^{3,8}$ However, radiologic evaluation often detects lateral root resorption on the pressure side of the moved tooth at the level of the knife-edge bone crest of an atrophic ridge, ${ }^{3,7,8}$ as shown in Figure 15. The prevalence of lateral root resorption is $40.6 \%$; the mean depth of the lesion was $0.7 \mathrm{~mm}(\mathrm{SD} \pm 0.3)$ and mean length was $2.3 \mathrm{~mm}(\mathrm{SD} \pm 0.6) .{ }^{3}$ In another study, lateral root resorption was found in almost all orthodontically treated teeth. ${ }^{7}$ The root resorption was independent of periodontal parameters such as plaque index or gingival index, but it was directly related to orthodontic force. ${ }^{8}$ An orthodontic load induces inflammation in the periodontium, particularly within the periodontal ligament (PDL). ${ }^{14}$ This reaction is necessary for tooth movement but it may also be associated with unwanted side effects, such as root resorption. When a tooth is moved distally, stress can induce necrosis in the PDL on the distal side of the tooth. ${ }^{15}$ Stress levels exceeding the necrotic threshold are associated with root resorption. ${ }^{16,17}$ In the edentulous area, the presence of thin, predominantly compact bone at the crestal site produces the most accumulated pressure (stress) as a tooth starts to move, thereby contributing to lateral root resorption. ${ }^{7}$ For the present patient, only minor lateral root resorption was observed in the crestal area (Fig. 15), which may be related to the light continuous force ( $20 z$ or $26.3 \mathrm{cN}$ per side) that was used to translate ${ }^{\# 20}$ distally. A serendipitous benefit associated with reduced PDL stress was rapid translation ( $1 \mathrm{~mm} /$ per month) of tooth \# 20 . This is twice as fast as initial molar movement and three times faster than sustained molar root movement in the posterior quadrant. ${ }^{18}$ The relatively rapid premolar translation for the present patient suggests the levels of stress in the PDL were relatively low resulting in continuous tooth movement that was relatively free of pressure necrosis. The force utilized was $26.3 \mathrm{cN}$ on the buccal and lingual surfaces for a total of $<57 \mathrm{cN}$, which is a much smaller load than typical space closure loops $(3 \mathrm{~N}) .{ }^{18}$ Another factor in accelerating the rate of tooth 
movement is the root morphology. A single rooted tooth like a mandibular premolar moves faster than a molar because the trailing root of a molar must resorb the dense bone formed by the leading root. ${ }^{18}$ Translation of molars is another option when considering the orthodontic formation of an implant space. Molars move slower and form denser bone, ${ }^{18}$ but there are no documented reports for implants placed in molar generated sites.

Previous studies have noted repair of root resorption after the termination of active tooth movement. ${ }^{7,19,20}$ Following tooth movement, areas of tooth resorption are repaired by deposition of new cementum. ${ }^{19}$ This reparative process is time dependent and is less responsive following the application of heavy orthodontic force. ${ }^{19}$ All considered, the continuous light force minimized PDL necrosis, and the long retention time (6 months) may have enhanced cementum repair. Those were probably the principal factors resulting in minimal lateral root resorption, despite root translation to prepare a $7 \mathrm{~mm}$ wide implant site in the posterior mandible (Fig. 8).

Orthodontically-produced potential implant sites are decreased $11 \%$ in the buccolingual dimension at the end of space opening mechanics and the atrophy progresses to $15 \%$ one year later. ${ }^{7}$ For the present patient, a similar result was observed despite placing a completely submerged implant with $1.5 \mathrm{~mm}$ of buccal bone thickness in the orthodontically generated space (Fig. 17-j). There was about $3 \mathrm{~mm}$ of crestal bone loss on the buccal surface when the implant was uncovered (Fig. 22-b). This phenomenon is uncommon when an implant is placed in a mature osseous site. However, for the present patient there was another variable: relapse of the space in width after the implant was placed and subsequent orthodontics to recover the space for an implantsupported prosthesis. It appears that much of the bone, that was resorbed when the implant was uncovered, was related to the relapse incident. This clinical experience indicates that retention of implant sites created with orthodontics is very important, both before and after placing the implants. It is unwise to use removable retainers because the patients may not cooperate. Thus, fixed retainers are indicated until the prosthesis is delivered.

Some crestal bone resorption is common when an implant is restored and is in contact with the oral environment. ${ }^{21}$ This phenomenon is often a biologic width violation: about $3 \mathrm{~mm}$ of soft tissue is required between the cervical margin of the crown and the alveolar bone crest. ${ }^{22}$ On average, it is found that the cervical height of the bone contact be adjusted to 1.5 to $2.0 \mathrm{~mm}$ below the implant shoulder when the implant is exposed, anticipating that there will be additional bone loss, which will hopefully result in the desired $3 \mathrm{~mm}$ biologic width. ${ }^{23}$ When an implant is completely buried inside the alveolar process the bone is usually still covering the implant when it is uncovered. However, for the present patient, the bone loss was found before the implant was exposed to the oral environment. As previously discussed this probably related to the immaturity of the bone at the time the implant was placed, but there was the added challenge of tooth \# 20 relapsing back into the space after the implant was placed. Moving \#20 back into position was expected to generate new bone on the distal surface of the implant, but if the distobuccal bone defect on the implant had already occurred, there may have been no subsequent bone formation to refill the space. The relapse of tooth \#20 probably resulted in resorption of all or most of the bone on the distal of the implant, and thereby contributed to the osseous dehiscence on the LL implant when it was uncovered.

The alveolar process is a tooth dependent tissue that is induced from competent neural crest cells. It develops and adapts in conjunction with the eruption or movement of the teeth. ${ }^{24,25}$ There are fundamental requirements for both tooth eruption and orthodontic tooth movement: (1) the mediation of biologically active soft tissues, i.e. the dental follicle for eruption and the periodontal ligament for tooth movement, (2) regulation of both bone modeling and remodeling, ${ }^{25}$ and (3) bone turnover (remodeling) that is temporarily and spatially regulated to facilitate specific translocations of teeth through alveolar bone. ${ }^{24}$ When orthodontic force is applied to a tooth, the strains (proportional to stress) created in the PDL initiate many biological processes that lead to cell proliferation and apoptosis. ${ }^{26}$ These competing processes control the cell population in the PDL and lead to 
different biological consequences for the PDL itself, as well as the alveolar bone and cementum. When orthodontic treatment is initiated, the PDL and supporting bone responds to the applied load, leading to bone resorption on the compression side, and deposition on the tension side of the PDL. ${ }^{27}$ However, there is also a great deal of modeling along the subperiosteal surfaces as well as internal remodeling of the alveolar process. ${ }^{28}$ These bone modeling and remodeling processes continue as the tooth moves relative to the basilar bone. The volume as well as the shape of the alveolar process is modified accordingly. Therefore, when the tooth is moved into the narrower edentulous ridge, there is initially a high stress concentration and lateral root resorption may occur where the PDL engages the dense knife-edge ridge (Fig. 15). The compressive loading of the edentulous ridge results in subperiosteal osteogenesis to increase the ridge dimension of the site into which the tooth is moved (Fig. 13). ${ }^{28}$ In contrast, when a tooth movement results in an edentulous ridge, the alveolar bone is no longer adequately loaded and undergoes disuse atrophy, which results in a decrease in the height of the ridge and loss of the buccal plate. ${ }^{2}$ This process continues until the residual bone in the implant site reach a steady-state equilibrium between the competitive, physiologic bone formation and resorption processes (Fig. 14). ${ }^{28}$

The progressive loss of bone in an orthodontically-generated edentulous area, is similar to the atrophy in height and width of the alveolar ridge following an extraction. 1,7,27 This healing process has more pronounced resorption on the buccal than on the lingual aspects of the ridge. Araujo and Lindhe ${ }^{2}$ in an experiment study in 12 mongrel dogs, found that the crestal region of the buccal hard tissue wall is made up exclusively of bundle bone that formed after the tooth had erupted. The lingual plate was composed of bundle bone and more mature lamellar bone that had undergone remodeling. Bundle bone is a variation of lamellar bone that is formed on bone appositional surfaces of ligament and tendon attachments, such as the PDL. ${ }^{28}$ It is characterized by vertical striations that are mineralized PDL fibers (Sharpey's Fibers) with bone formed around them. Araujo and Lindhe ${ }^{2}$ demonstrated that the reduced ridge dimension was more pronounced in the buccal and crestal aspects of the edentulous space, due to a high rate of bundle bone resorption. They noted there were two phases related to the postoperative bone atrophy: Phase 1. surgical trauma, avascular necrosis and wound healing resulted in loss of bundle bone and replacement with woven bone, and Phase 2. sustained resorption on the outer surface of both the buccal and lingual plates. Although Araujo and Lindhe ${ }^{2}$ had no explanation for the latter sustained bone loss, the physiologic mechanism appeared to be biomechanics. When bone is no longer internally loaded by the root of a tooth, there is disuse atrophy of the most peripheral aspects of the bone, alveolar crest and buccal plate. ${ }^{28}$ In effect, disuse atrophy tends to produce bone resorption at same time that implant wound healing and bone grafting procedures are enhancing bone formation. This competitive process appears to be an important factor for why bone defects tend to occur on the buccal (Fig.22-b,c) rather than the lingual. The buccal surface is the most peripheral aspect of a curved cortical bone surface, so it is most likely to be affected by disuse atrophy. ${ }^{28}$

\section{Conclusions}

Adult orthodontic patients often present with missing teeth and atrophic edentulous ridges. Assuming the adjacent teeth are periodontally healthy, a narrow ridge can be expanded with orthodontic tooth movement. The process is a distraction of the PDL to produce bundle bone: mineralized collagen bundles (Sharpey's fibers) within bone. Lateral root resorption is common when the leading surface of the root engages the dense cortical bone of the narrow atrophic ridge. Light forces for mandibular premolar translation were expected to reduce PDL necrosis, and the expression of lateral root resorption, but they also substantially increased the rate of tooth movement. When space is opened between teeth to create an implant site, the newly established ridge is predominantly immature bundle bone, and it begins to atrophy in height and buccal width as soon as it is formed. Ridge augmentation is recommended at the time of implant placement to compensate for the additional anticipated bone loss. A fixed retainer is strongly advised for space maintenance until the implant- 
supported prosthesis is completed. Despite bone and soft tissue problems in the implant sites, an excellent overall outcome was achieved, as evidenced by an ABO CRE score of 13 (supplementary worksheet 2).

\section{Acknowledgment}

Thanks to Dr. Angle Lee for correcting the cephalometric tracings and to Mr. Paul Head for proofreading this article.

\section{References}

1. Pietrokovski J \& Massler M. Alveolar ridge resorption following tooth extraction. J Prosthet Dent 1967;17:21-27.

2. Araujo MG, Lindhe J. Dimensional ridge alterations following tooth extraction. An experimental study in the dog. J Clin Periodontol 2005;32:212-218.

3. Diedrich PR, Fuhrmann RA, Wehrbein H, Erpenstein H. Distal movement of premolars to provide posterior abutments for missing molars. Am J Orthod Dentofacial Orthop 1996;109:355-360.

4. Chen ST, Buser D. Clinical and Esthetic outcome of implants placed in postextraction sites. Int J Oral Maxillofac Implants 2009;24(suppl):186-217.

5. Roberts WE. Adjunctive orthodontic therapy in adults over 50 years of age: clinical management of compensated, partially edentulous malocclusion. J Indiana Dent Assoc 1997;76(2):33-41

6. Roberts WE. Bone dynamics of osseointegration, ankylosis and tooth movement: tissue engineering. J Indiana Dent Assoc 1999;78(3):24-32.

7. Lindskog-Stokland B, Hansen K, Ekestubbe A, Wennstrom JL. Orthdontic tooth movement into edentulous ridge areas-a case series. Eur J Orthod 2013;35(3):277-285.

8. Giannopoulou C, Dudic A, Montet X, Kiliaridis S, Mombelli A. Periodontal parameters and cervical root resorption during orthodontic tooth movement. J Clin Periodontol 2008;35(6):501-506.

9. Kokich VG, Kokich VO. Congenitally missing mandibular second premolars: clinical options. Am J Orthod Dentofacial Orthop 2006;130(4):437-44.

10. Cangialosi TJ, Riolo ML, Owens SE Jr, Dykhouse VJ, Moffitt AH, Grubb JE, Greco PM, English JD, James RD. The ABO discrepancy index: A measure of case complexity. Am J Orthod Dentofacial Orthop 2004;125:270-8.

11. Chang $\mathrm{CH}$. The $2 \mathrm{~B}-3 \mathrm{D}$ rule for implant planning, placement and restoration. Int $\mathrm{J}$ Orthod Implantol 2012;27:96-101.

12. Femminella B, laconi MC, Di Tullio M, Romano L, Sinjari B, D'Arcangelo C. Clinical comparison of platelet-rich fibrin and a gelatin sponge in the management of palatal wounds after epithelialized free gingival graft harvest: A randomized clinical trial. J Periodontol 2016;87(2):103-113.

13. Casko JS, Vaden JL, Kokich VG, Damone J, James RD, Cangialosi TJ, Riolo ML, Owens SE Jr, Bills ED. Objective grading system for dental casts and panoramic radiographs. Am J Orthod Dentofacial Orthop 1998;114(5):589-99.

14. Krishnan V, Davidovitch Z. Cellular, molecular, and tissue-level reactions to orthodontic force. Am J Orthod Dentofacial Orthop 2006;129:469. e. I-469, e. 32. 
15. Roberts WE, Viecilli RF, Chang C, Katona TR, Paydar NH. Biology of biomechanics: finite element analysis of a statically determinate system to rotate the occlusal plane for correction of skeletal Class III malocclusion. Am J Orthod Dentofac Orthop 2015;148:943-955.

16. Viecilli RF, Kar-Kuri MH, Variable J, Budiman A, Janal M. Effectod of initial stresses and time on orthodontic external root resorption. J Dent Res 2013;92(4):346-51.

17. Viecilli RF, Katona TR, Chen J, Hartsfield JK, Roberts WE. Orthodontic Mechanotransduction and the Role of the P2X7 Receptor. Am J Orthod Dentofac Orthop 2009;135:694. e. 1-16; discussion 694-5

18. Roberts WE, Arbuckle GR, Analoui M. Rate of mesial translation of mandibular molars using implantanchored mechanics. Angle Orthod 1996;66(5):331-338.

19. Cheng LL, Turk T, Elekdag-Turk S, Jones AS, Yu Y, Darendeliler MA. Repair of root resorption 4 and 8 weeks after application of continuous light and heavy forces on premolars for 4 weeks: a histology study. Am J Orthod Dentofacial Orthop 2010;138:727-734.

20. Jimenez-Pellegrin C, Arana-Chavez VE. Root resorption repair in mandibular first premolars after rotation. A transmission electron microscopy analysis combined with immunolabeling of osteopontin. Am J Orthod Dentofacial Orthop 2007;132:230-236.

21. Grunder U, Gracis S, Capelli M. Influence of the 3D bone to implant relationship on esthetics. Int J Periodontics Restorative Dent 2005 Apr;25(2):113-119.

22. Calvo-Guirado JL, López-López PJ, Pérez-Albacete Martínez C, Javed F, Granero-Marín JM, Maté Sánchez de Val JE, Ramírez Fernández MP. Peri-implant bone loss clinical and radiographic evaluation around rough neck and microthread implants: a 5-year study. Clin Oral Implants Res 2016 Jan 7. doi: 10.1111/clr.12775. [Epub ahead of print]

23. Adell R, Lekholm U, Rockler B, Branemark PI. A 15-year study on osseointegrated implants in the treatment of the edentulous jaw. Int J Oral Surg 1981;10:387-416.

24. Wise GE, King GJ. Mechanism of tooth eruption and orthodontic tooth movement. J Dent Res 2008;87(5):414-434.

25. Roberts WE, Goodwin WC, Heiner SR. Cellular response to orthodontic force. Dental Clinics of North America 1981;25(1):3-17.

26. Roberts WE, Chase DC. Kinetics of cell proliferation and migration associated with orthodonticallyinduced osteogenesis. J Dent Res 1981;60:174-181.

27. King GJ, Keeling SD, Wronski TJ. Histomorphometric study of alveolar bone turnover in orthodontic tooth movement. Bone 1991;12:401-409.

28. Roberts WE. Bone physiology, metabolism and biomechanics in orthodontic practice. In: Orthodontics: Current Principles and Techniques, Chapter 10, $5^{\text {th }}$ ed. Graber LW, Vanarsdall RL Jr., Vig KWL (Eds) Mosby, St Louis: Elsevier; 2012. pp. 287-343.

29. Schropp L, Wenzel A, Kostopoulos L, Karring T. Bone healing and soft tissue contour changes following single-tooth extraction: a clinical and radiographic 12-month prospect study. Int J Periodontics Restorative Dent 2003;23(4): 313-323.

\section{Figure Legends}

Fig. 1: Pre-treatment facial photograph.

Fig. 2: Pre-treatment intraoral photographs.

Fig. 3: Pre-treatment dental models (casts) 
Fig. 4: Post-treatment facial photographs

Fig. 5: Post-treatment intraoral photographs

Fig. 6: Post-treatment study models (casts)

Fig. 7: Pre-treatment cephalometric (above) and panoramic (below) radiographs

Fig. 8: Post-treatment cephalometric (above) and panoramic (below) radiographs

Fig. 9: Superimposed cephalometric tracings show protraction and uprighting of tooth \#17, and retraction of the lower incisors.

Fig. 10:

$0 \mathrm{M}$. The irregular dentition is shown at the start of active treatment.

7M. Seven months into active treatment the lower left segment is being aligned and the edentulous space is decreased. Note the lingual elastic chain between the left molar and second premolar. See text for details $16 \mathrm{M}$. At sixteen months the initial alignment is complete.

Fig. 11: The lower left space (\#19) was reduced and the lower right space (\#30) was maintained with a fixed temporary prosthesis.

Fig. 12: The edentulous ridge for the \#19 space was very narrow so \#20 was moved distally with an open coil spring (arrow) on the buccal surface, between \#20 and \#21, and buttons with power chains on the lingual surface.

Fig. 13: As shown at 21 months $(21 \mathrm{M})$, tooth $\# 20$ was moved into the edentulous ridge in the area of tooth $\# 19$.

Fig. 14: At 32 months (32M) into treatment : (a) \#20 was moved to the position of \#19 with 7 months of distal translation mechanics, and (b) from the occlusal view the width of the ridge in the implant site was around $7 \mathrm{~mm}$ (including soft tissue).

Fig. 15: A periapical radiograph documents that $\# 20$ was translated distally to contact $\# 17$ and a small area of lateral root resorption was noted near the crestal area of the alveolar process (yellow arrow).

Fig. 16: Pre-treatment (Pre-Tx) or zero months of active treatment (OM) mesial-in rotations are noted for both lateral incisors. Interproximal reduction was performed on \#7-10 to create space to correct the rotations as shown in the post-treatment (Post-Tx) occlusal view at 38 months (38M).

Fig. 17: The steps involved for placing an implant in the orthodontically generated space: (a) \#20 was moved distally to produce a space between the lower left premolars, (b) occlusal view of the prepared implant site, (c) a horizontal crestal incision and two vertical releasing incisions were made $1.5 \mathrm{~mm}$ away from adjacent teeth, (d) occlusal view of the osseous ridge, (e) ridge width was about $6 \mathrm{~mm}$, (f) a surgical stent showed the cervical position of the proposed crown was only $1 \mathrm{~mm}$ from the bone crest, $(\mathrm{g})$ crestal bone was removed with a round bur, (h) $3 \mathrm{~mm}$ clearance from cervical contour of surgical stent to bone crest was created to achieve the necessary biologic width, (i) a guide pin showed the angulation of the osteotomy, (j) a $3.5 \times 10 \mathrm{~mm}$ implant was placed completely within the bone, $(\mathrm{k})$ a cover screw was seated, and $(\mathrm{l})$ primary closure of the wound was achieved with 5-0 Nylon.

Fig. 18: (a) Occlusal view of the edentulous ridge in the area of \#30. (b) A horizontal crestal incision and two vertical incisions were made. (c) The edentulous ridge is exposed. (d) Guide pin was placed to check the path of insertion relative to the osteotomy orientation. (e) A $3.5 \times 10 \mathrm{~mm}$ implant was placed. (f) There was exposed implant interface (rough surface). (g) The healing abutment was connected and a particulate bone graft (FDBA+Bio-Oss $®$ ) was placed on the buccal side to cover the exposed implant surface. (h) The wound was primarily closed with 5-0 Nylon.

Fig. 19: (a) The \#30 space was maintained with the fixed appliances, and a plastic sheath was placed on the archwire to control soft tissue irritation.

(b) An open-coil spring on the archwire maintained the orthodontically generated implant site in the \#20 space on the left side.

Fig. 20: One month after the fixed appliances were removed, tooth \#20 relapsed into the site where the implant was healing.

Fig. 21: (a) B-0 marks the start of retreatment to correct the relapse. Lower fixed appliances were reinstalled and power chains with an open coil spring was used to move \#20 back to the desired position. (b) B-9 is an occlusal view of the completed lower arch at the 3 mo recall visit after treatment was completed, which was 
9 months following the start of retreatment phase. A fixed retainer was bonded on the buccal surface of \#17 and 20 (upper right) to avoid any further relapse.

Fig. 22: The lower left implant (area \#20) was uncovered, surgically revised and restored. (a) A horizontal crestal and two vertical incisions were made. (b) After flap reflection, $>2 \mathrm{~mm}$ of rough implant surface was exposed. (c) A healing abutment was installed. (d) A particulate bone graft composed of FDBA and BioOss ${ }^{\circledR}$ was placed over the exposed implant interface. (e) Primary closure of the wound was achieved. (f) Two months later an implant-supported prosthesis was fabricated to restore occlusion (center).

Fig. 23: There was a deep concave area on the cervical portion of lower right implant-supported prosthesis (area \#30) superior to the buccal vestibule. In addition, there was only a $0.5 \mathrm{~mm}$ width of keratinized gingiva. Although the tissue was healthy, the recessed interproximal areas were an irritating food trap for the patient.

Fig. 24: (a) Preoperatively, a slightly more anterior view demonstrated that the recessed area on the distal of the implant-supported prosthesis was the most pronounced (center). (b) A horizontal incision was made apical to the attached gingiva from teeth \#29-31 and a partial thickness flap was reflected. (c) A 5x15mm free gingival graft was harvested from right palatal mucosa. (d) The donor site was covered with platelet rich fibrin (PRF) to enhance wound healing and was cross sutured with 5-0 Nylon. (e) The free gingiva graft was sutured to the recipient site with 5-0 nylon. (f) A Coe-Pak® dressing was placed to protect the graft and prevent the buccal flap from competing with the graft for attachment to the inferior portion of the recipient bed.

Fig. 25: Following completion of the postoperative healing, the free gingiva graft increased the width of the attached tissue (left), and corrected the contour for food defection into the buccal fold during mastication.

Table 1: Cephalometric summary 